\title{
SOME RESULTS ON THE SPECTRAL RADII OF TREES, UNICYCLIC, AND BICYCLIC GRAPHS*
}

\author{
MUHUO LIU ${ }^{\dagger}$ AND BOLIAN LIU
}

\begin{abstract}
Let $\Delta(G), \Delta$ for short, be the maximum degree of a graph $G$. In this paper, trees (resp., unicyclic graphs and bicyclic graphs), which attain the first and the second largest spectral radius with respect to the adjacency matrix in the class of trees (resp., unicyclic graphs and bicyclic graphs) with $n$ vertices and the maximum degree $\Delta$, where $\Delta \geq \frac{n+1}{2}$ (resp., $\Delta \geq \frac{n}{2}+1$ and $\Delta \geq \frac{n+3}{2}$ ) are determined. Moreover, it is shown that the spectral radius of a unicyclic graph $U$ (resp., a bicyclic graph $B$ ) on $n$ vertices strictly increases with its maximum degree when $\Delta(U) \geq \frac{1}{9}(1+\sqrt{6 n+10})^{2}$ (resp., $\left.\Delta(B) \geq \frac{1}{9}(2+\sqrt{6 n+28})^{2}\right)$.
\end{abstract}

Key words. Spectral radius, Maximum degree, Unicyclic graph, Bicyclic graph.

AMS subject classifications. 05C35, 15A48, 05C50.

1. Introduction. Throughout the paper, $G=(V, E)$ is a connected undirected simple graph with $|V|=n$ and $|E|=m$. If $m=n+c-1$, then $G$ is called a $c$-cyclic graph. In particular, if $c=0,1$ or 2 , then $G$ is called a tree, a unicyclic graph or a bicyclic graph, respectively.

Let $N(v)$ indicate the set of neighbors of vertex $v$. Then, $d(v)=|N(v)|$ is called the degree of $v$. Let $\Delta(G), \Delta$ for short, be the maximum degree of $G$. We use the notations $\mathbb{T}_{n}^{\Delta}, \mathbb{U}_{n}^{\Delta}$ and $\mathbb{B}_{n}^{\Delta}$ to denote the class of trees, unicyclic graphs and bicyclic graphs with $n$ vertices and the maximum degree $\Delta$, respectively.

If $d_{i}=d\left(v_{i}\right)$ for $i=1,2, \ldots, n$, then we call the sequence $\pi(G)=\left(d_{1}, d_{2}, \ldots, d_{n}\right)$ the degree sequence of $G$. In the following discussion, we enumerate the degrees in non-increasing order, i.e., $d_{1} \geq d_{2} \geq \cdots \geq d_{n}$. When more than one graph is under discussion, we may write $d_{i}(G)$ instead of $d_{i}$. We use $\Gamma(\pi)$ to denote the class of connected graphs with the degree sequence $\pi$, where $\pi=\left(d_{1}, d_{2}, \ldots, d_{n}\right)$.

${ }^{*}$ Received by the editors on May 21, 2011. Accepted for publication on March 24, 2012. Handling Editor: João Filipe Queiro. This work was supported by the Foundation for Distinguished Young Talents in Higher Education of Guangdong, China (no. LYM10039) and NNSF of China (no. 11071088).

${ }^{\dagger}$ Institute of Mathematics, School of Mathematical Science Nanjing Normal University, Nanjing, 210046, P.R. China; Department of Mathematics, South China Agricultural University, Guangzhou, 510642, P.R. China; School of Mathematic Science, South China Normal University, Guangzhou, 510631, P.R. China (liumuhuo@163.com).

${ }_{\ddagger}^{\ddagger}$ School of Mathematic Science, South China Normal University, Guangzhou, 510631, P.R. China (liubl@scnu.edu.cn). 
Let $A(G)$ be the adjacency matrix of $G$. By the Perron-Frobenius Theorem of non-negative matrices, the largest eigenvalue of $A(G)$ is equal to the spectral radius of $A(G)$. We simply call the latter the spectral radius of $G$ and denote it by $\rho(G)$. The characteristic polynomial $\operatorname{det}(x I-A(G))$ of $A(G)$ is referred to as the characteristic polynomial of $G$ and is denoted by $\Phi(G, x)$. Thus, $\rho(G)$ equals to the maximum root of $\Phi(G, x)=0$.

When $G$ is connected, by the Perron-Frobenius Theorem of non-negative matrices, there exists a unique positive unit eigenvector corresponding to $\rho(G)$. In the following, we use $f=\left(f\left(v_{1}\right), \ldots, f\left(v_{n}\right)\right)^{T}$ to be the unique positive unit eigenvector corresponding to $\rho(G)$, and we call $f$ the Perron vector of $G$. Moreover, $f\left(v_{i}\right)$ is also called the $\rho$-weight of the $i$ th vertex (with respect to $f$ ).

In 1981, Cvetković [3] indicated 12 directions in further investigations of graph spectra, one of which is classifying and ordering graphs. Hence, ordering graphs with various properties by their spectra, becomes an attractive topic (see [5, 8, 9, 10]). In this line, Lin and Guo [5] had proved that:

THEOREM 1.1. 5] Let $\Delta$ and $n$ be two fixed positive integers.

(i) If $\Delta \geq\left\lceil\frac{n}{2}\right\rceil$, then $H_{1}(n, \Delta)$ (see Fig. 2.1) is the unique tree with the largest spectral radius among the trees in $\mathbb{T}_{n}^{\Delta}$.

(ii) For any two trees $T$ and $T^{\prime}$ on $n \geq 4$ vertices, if $\Delta(T) \geq\left\lceil\frac{2 n}{3}\right\rceil-1$ and $\Delta(T)>\Delta\left(T^{\prime}\right)$, then $\rho(T)>\rho\left(T^{\prime}\right)$.

Recently, a similar result was obtained for unicyclic graphs, that is:

THEOREM 1.2. 10] Let $\Delta$ and $n$ be two fixed positive integers.

(i) If $\Delta \geq \frac{n}{2}+1$, then $F_{1}(n, \Delta)$ (see Fig. 3.1) is the unique unicyclic graph with the largest spectral radius among the unicyclic graphs in $\mathbb{U}_{n}^{\Delta}$.

(ii) For any two unicyclic graphs $U$ and $U^{\prime}$ on $n \geq 30$ vertices, if $\Delta(U) \geq\left\lceil\frac{7 n}{9}\right\rceil+1$ and $\Delta(U)>\Delta\left(U^{\prime}\right)$, then $\rho(U)>\rho\left(U^{\prime}\right)$.

Moreover, Yuan et al. also considered the corresponding problem for bicyclic graphs and proved the following:

THEOREM 1.3. 9] Let $\Delta$ and $n$ be two fixed positive integers.

(i) If $\Delta \geq \frac{n+3}{2}$, then $W_{1}(n, \Delta)$ (see Fig. 4.1) is the unique bicyclic graph with the largest spectral radius among the bicyclic graphs in $\mathbb{B}_{n}^{\Delta}$.

(ii) For any two bicyclic graphs $B$ and $B^{\prime}$ on $n$ vertices, if $\Delta(B) \geq\left\lceil\frac{7 n}{9}\right\rceil+9$ and $\Delta(B)>\Delta\left(B^{\prime}\right)$, then $\rho(B)>\rho\left(B^{\prime}\right)$. 
In this paper, we shall establish the following results.

THEOREM 1.4. Let $\Delta$ and $n$ be two fixed positive integers. If $\frac{n+1}{2} \leq \Delta \leq$ $n-3$ and $T \in \mathbb{T}_{n}^{\Delta} \backslash\left\{H_{1}(n, \Delta), H_{2}(n, \Delta)\right\}$ (see Fig 2.1), then $\rho(T)<\rho\left(H_{2}(n, \Delta)\right)<$ $\rho\left(H_{1}(n, \Delta)\right)$.

THEOREM 1.5. Let $\Delta$ and $n$ be two fixed positive integers.

(i) If $\frac{n}{2}+1 \leq \Delta \leq n-3$ and $U \in \mathbb{U}_{n}^{\Delta} \backslash\left\{F_{1}(n, \Delta), F_{2}(n, \Delta)\right\}$ (see Fig. 3.1), then $\rho(U)<\rho\left(F_{2}(n, \Delta)\right)<\rho\left(F_{1}(n, \Delta)\right)$.

(ii) For any two unicyclic graphs $U$ and $U^{\prime}$ on $n$ vertices, if $\Delta(U) \geq$ $\frac{1}{9}(1+\sqrt{6 n+10})^{2}$ and $\Delta(U)>\Delta\left(U^{\prime}\right)$, then $\rho(U)>\rho\left(U^{\prime}\right)$.

ThEOREM 1.6. Let $\Delta$ and $n$ be two fixed positive integers.

(i) If $\frac{n+3}{2} \leq \Delta \leq n-2$, and $B \in \mathbb{B}_{n}^{\Delta} \backslash\left\{W_{1}(n, \Delta), W_{2}(n, \Delta)\right\}$ (see Fig. 4.1), then $\rho(B)<\rho\left(W_{2}(n, \Delta)\right)<\rho\left(W_{1}(n, \Delta)\right)$.

(ii) For any two bicyclic graphs $B$ and $B^{\prime}$ on $n$ vertices, if $\Delta(B) \geq$ $\frac{1}{9}(2+\sqrt{6 n+28})^{2}$ and $\Delta(B)>\Delta\left(B^{\prime}\right)$, then $\rho(B)>\rho\left(B^{\prime}\right)$.

If $n \geq 30$, then $\frac{1}{9}(1+\sqrt{6 n+10})^{2}<\left\lceil\frac{7 n}{9}\right\rceil+1$. Moreover, it is easily checked that $\frac{1}{9}(2+\sqrt{6 n+28})^{2}<\left\lceil\frac{7 n}{9}\right\rceil+9$. Thus, we can conclude that

REMark 1.7. Theorem 1.4 extends the result of Theorem 1.1 (i), Theorem 1.5 improves the result of Theorem 1.2 , and Theorem 1.6 improves the result of Theorem 1.3

2. The proof of Theorem 1.4. Let $G-u$ (resp., $G-u v$ ) be the graph obtained from $G$ by deleting the vertex $u \in V(G)$ (respectively, the edge $u v \in E(G)$ ). Similarly, denote by $G+u v$ the graph obtained from $G$ by adding an edge $u v \notin E(G)$.

LEMMA 2.1. [8] Let $u, v$ be two vertices of the connected graph $G$, and $w_{1}, w_{2}$, $\ldots, w_{k}(1 \leq k \leq d(v))$ be some vertices of $N(v) \backslash N(u)$. Let $G^{\prime}=G+u w_{1}+\cdots+$ $u w_{k}-v w_{1}-\cdots-v w_{k}$. Suppose that $f$ is the Perron vector of $G$. If $f(u) \geq f(v)$, then $\rho\left(G^{\prime}\right)>\rho(G)$.

Lemma 2.2. 2] Let $G=(V, E)$ be a connected graph such that $u_{1} v_{1} \in E, u_{2} v_{2} \in$ $E, v_{1} v_{2} \notin E$, and $u_{1} u_{2} \notin E$. Let $G^{\prime}=G+v_{1} v_{2}+u_{1} u_{2}-u_{1} v_{1}-u_{2} v_{2}$. Suppose that $f$ is the Perron vector of $G$. If $f\left(v_{1}\right) \geq f\left(u_{2}\right)$ and $f\left(v_{2}\right) \geq f\left(u_{1}\right)$, then $\rho\left(G^{\prime}\right) \geq \rho(G)$, where the equality holds if and only if $f\left(v_{1}\right)=f\left(u_{2}\right)$ and $f\left(v_{2}\right)=f\left(u_{1}\right)$.

Let $G$ be a connected graph, and $u v \in E(G)$. The graph $G_{u, v}$ is obtained from $G$ by subdividing the edge $u v$, i.e., adding a new vertex $w$ and edges $w u, w v$ in $G-u v$. An internal path, say $v_{1} v_{2} \cdots v_{s+1}(s \geq 1)$, is a path joining $v_{1}$ and $v_{s+1}$ (which need not be distinct) such that $v_{1}$ and $v_{s+1}$ have degree greater than 2 , while all other 
vertices $v_{2}, \ldots, v_{s}$ are of degree 2 . Let $W_{n}$ be the tree obtained from a path $P_{n-4}$ by attaching two pendant vertices to each pendant vertex of $P_{n-4}$, respectively.

LEMma 2.3. 4] Let uv be an edge of the connected graph $G$ on $n$ vertices. If uv belongs to an internal path of $G$, and $G \neq W_{n}$, then $\rho(G)>\rho\left(G_{u, v}\right)$.

LEMMA 2.4. Let $G$ be a graph with the largest spectral radius in $\Gamma(\pi)$, where $\pi=\pi(G)$, and $u, v \in V(G)$. Suppose that $f$ is the Perron vector of $G$. If $d(u)>d(v)$, then $f(u)>f(v)$.

Proof. Suppose to the contrary, there exist $u, v \in V(G)$ such that $d(u)>d(v)$, but $f(u) \leq f(v)$. Let $d(u)-d(v)=k$. Let $P_{u v}$ be the shortest path from $u$ to $v$. Then, there must exist $k$ vertices, say $w_{1}, \ldots, w_{k}$, such that $w_{1}, \ldots, w_{k} \in N(u) \backslash N(v)$ and $w_{1}, \ldots, w_{k} \notin V\left(P_{u v}\right)$. Let $G_{1}=G-u w_{1}-\cdots-u w_{k}+v w_{1}+\cdots+v w_{k}$. By Lemma 2.1. $\rho(G)<\rho\left(G_{1}\right)$. But $G_{1} \in \Gamma(\pi)$, so we arrive at a contradiction. Thus, $f(u)>f(v)$.

Denote by $\mathbb{S}(n, c ; \Delta)$ the set of connected $c$-cyclic graphs on $n$ vertices with the maximum degree $\Delta$.

LEMma 2.5. 7] If $\Delta \leq n-2$, then

$$
\max \{\rho(G): G \in \mathbb{S}(n, c ; \Delta)\}<\max \{\rho(G): G \in \mathbb{S}(n, c ; \Delta+1)\}
$$

Lemma 2.6. 7] Let $G=(V, E)$ be a graph of $\mathbb{S}(n, c ; \Delta)$, where $\Delta \geq \frac{n+c+1}{2}$. If $d(u)=\Delta$, then $u$ is the unique vertex with degree $\Delta$.

Let $\mathbb{T}(n, \Delta, d)$ be the set of trees on $n$ vertices with the maximum degree $\Delta$ and the second maximum degree $d$.

LEMmA 2.7. If $\Delta-d \geq 2$ and $d \leq n-\Delta-1$, then

$$
\max \{\rho(T): T \in \mathbb{T}(n, \Delta, d)\}<\max \{\rho(T): T \in \mathbb{T}(n, \Delta, d+1)\}
$$

Proof. Let $T_{1}$ be a tree with the largest spectral radius in $\mathbb{T}(n, \Delta, d)$. Then, $T_{1}$ also has the largest spectral radius in $\Gamma(\pi)$, where $\pi=\pi\left(T_{1}\right)$. Since $\Delta-d \geq 2$, there exists a unique vertex, say $u$, such that $d(u)=\Delta$. Let $f$ be the Perron vector of $T_{1}$. Let $v$ be a vertex of $T_{1}$ such that $f(v)=\max \{f(w): d(w)=d\}$. There are two cases to be considered.

Case 1. $u v \notin E\left(T_{1}\right)$.

Let $P_{u v}$ be the shortest path of $T_{1}$ from $u$ to $v$, and let $x$ be the vertex in $P_{u v}$ 
adjacent to $u$. Let $T^{\prime}=T_{1}+u v-u x$. Then, $T^{\prime} \in \mathbb{T}(n, \Delta, d+1)$. By Lemma 2.4 and the choice of $v, f(v) \geq f(x)$. By Lemma 2.1, it follows that $\rho\left(T^{\prime}\right)>\rho\left(T_{1}\right)$.

Case 2. $u v \in E\left(T_{1}\right)$.

Since $T_{1} \in \mathbb{T}(n, \Delta, d), d \leq n-\Delta-1$ and $u, v$ are adjacent, we have $V(T) \neq$ $N(u) \cup N(v) \cup\{u, v\}=N(u) \cup N(v)$. So there exists a vertex $z$, different from $u, v$ and not adjacent to $v$, which is adjacent to $x$ where $x \in N(u) \cup N(v)$. By Lemma 2.4 and the choice of $v$, we have $f(v) \geq f(x)$. Let $T^{\prime}=T_{1}+v z-x z$. Then, $T^{\prime} \in \mathbb{T}(n, \Delta, d+1)$ and by Lemma 2.1 we have $\rho\left(T^{\prime}\right)>\rho\left(T_{1}\right)$.

Remark 2.8. The condition " $d \leq n-\Delta-1$ " of Lemma 2.7 is necessary. Otherwise, if $d \geq n-\Delta$, then $\Delta+d+1 \geq n+1$, that is, there are two vertices $u$ and $v$ such that $|N(u) \cap N(v)| \geq 1$ if they are adjacent and $|N(u) \cap N(v)| \geq 2$ otherwise. In both cases we obtain at least one cycle.
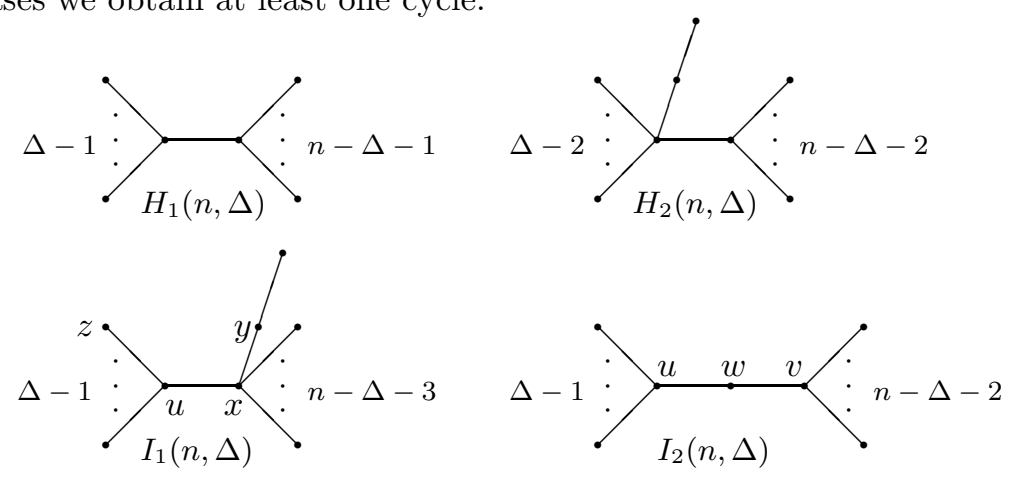

FIG. 2.1. The trees $H_{1}(n, \Delta), H_{2}(n, \Delta), I_{1}(n, \Delta), I_{2}(n, \Delta)$.

In the following, let $H_{1}(n, \Delta), H_{2}(n, \Delta), I_{1}(n, \Delta)$ and $I_{2}(n, \Delta)$ be the trees with $n$ vertices and the maximum degree $\Delta \geq \frac{n+1}{2}$ as shown in Fig. 2.1.

Proof of Theorem 1.4. It is easily checked that $H_{1}(n, \Delta)$ is the unique tree in $\mathbb{T}(n, \Delta, n-\Delta)$. Note that $d_{2}\left(H_{2}(n, \Delta)\right)=n-\Delta-1<n-\Delta=d_{2}\left(H_{1}(n, \Delta)\right)$, and $\Delta-(n-\Delta-1)=2 \Delta+1-n \geq 2$. Then, $\rho\left(H_{2}(n, \Delta)\right)<\rho\left(H_{1}(n, \Delta)\right)$ follows from Lemma 2.7. Suppose that the degree sequence of $T$ is $(a)=\left(d_{1}, d_{2}, d_{3}, \ldots, d_{n}\right)$. Since $T \in \mathbb{T}_{n}^{\Delta}, d_{1}=\Delta$. There are two cases to be considered.

Case 1. $d_{2}=n-\Delta-1$.

Note that $T \neq H_{2}(n, \Delta)$. Then, $T$ is isomorphic with $I_{1}(n, \Delta)$ or $I_{2}(n, \Delta)$. Let $u$, $v, w$ be the vertices of $I_{2}(n, \Delta)$ as shown in Fig. 2.1. Let $T^{\prime}=I_{2}(n, \Delta)-u w-v w+u v$. Since $\Delta \geq \frac{n+1}{2}, I_{2}(n, \Delta) \nsucc W_{n}$. By Lemma 2.3, $\rho\left(I_{2}(n, \Delta)\right)<\rho\left(T^{\prime}-w\right)=\rho\left(T^{\prime}\right)$. Note that $T^{\prime}$ is a proper subgraph of $I_{1}(n, \Delta)$. Thus, $\rho\left(T^{\prime}\right)<\rho\left(I_{1}(n, \Delta)\right)$, and hence, $\rho\left(I_{2}(n, \Delta)\right)<\rho\left(I_{1}(n, \Delta)\right)$. 
Now we shall prove that $\rho\left(I_{1}(n, \Delta)\right)<\rho\left(H_{2}(n, \Delta)\right)$. Assume to the contrary that $\rho\left(H_{2}(n, \Delta)\right) \leq \rho\left(I_{1}(n, \Delta)\right)$. Then, $I_{1}(n, \Delta)$ has the largest spectral radius in $\mathbb{T}(n, \Delta, n$ $-\Delta-1)$ because $I_{1}(n, \Delta), I_{2}(n, \Delta)$ and $H_{2}(n, \Delta)$ are precisely all the trees in $\mathbb{T}(n, \Delta, n$ $-\Delta-1)$. Then, $I_{1}(n, \Delta)$ also has the largest spectral radius in $\Gamma(a)$. Since $\Delta \geq \frac{n+1}{2}$, by Lemma 2.6 there exists a unique vertex in $I_{1}(n, \Delta)$, say $u$, such that $d(u)=\Delta$. Let $f$ be the Perron vector of $I_{1}(n, \Delta)$, and $u, x, y, z$ be the vertices of $I_{1}(n, \Delta)$ as shown in Fig. 2.1. By Lemma 2.4, $f(u)>f(x)$ and $f(y)>f(z)$ because $d(u)>d(x)$ and $d(y)>d(z)$. Let $T^{*}=I_{1}(n, \Delta)+u y+x z-u z-x y$. Then, $\rho\left(I_{1}(n, \Delta)\right)<\rho\left(T^{*}\right)$ by Lemma 2.2. Note that $T^{*}=H_{2}(n, \Delta)$. It is a contradiction to the hypothesis. Thus, $\rho\left(I_{1}(n, \Delta)\right)<\rho\left(H_{2}(n, \Delta)\right)$.

Case 2. $d_{2} \leq n-\Delta-2$.

Note that $\Delta-d_{2} \geq \Delta-(n-\Delta-2)=2 \Delta+2-n>2$. By Lemma 2.7 and the proof of Case 1, it easily follows that $\rho(T)<\rho\left(H_{2}(n, \Delta)\right)$.

Combining the above arguments, we complete the proof. $\square$

3. The proof of Theorem 1.5. To characterize the graphs that have greatest spectral radii among all graphs in $\Gamma(\pi)$, Biyıkoğlu and Leydold [2] introduced an ordering of the vertices $v_{0}, \ldots, v_{n-1}$ of a graph $G$ on $n$ vertices by means of breadth-first search as follows: Select a vertex $v_{0} \in G$ and create a sorted list of vertices beginning with $v_{0}$; append all neighbors $v_{1}, \ldots, v_{d\left(v_{0}\right)}$ of $v_{0}$ sorted by decreasing degrees; then append all neighbors of $v_{1}$ that are not already in this list; continue recursively with $v_{2}, v_{3}, \ldots$ until all vertices of $G$ are processed. Such an ordering is called a spiral like ordering [2], or spiral like disposition [1, 6].

LEMMA 3.1. [1] Let $\pi=\left(d_{1}, d_{2}, \ldots, d_{n}\right)$ be a non-increasing degree sequence and $U_{M}$ be a unicyclic graph with the largest spectral radius in $\Gamma(\pi)$. Suppose that $\left\{v_{1}, \ldots, v_{n}\right\}$ are the vertices of $U_{M}$ with $d\left(v_{i}\right)=d_{i}$, then $v_{1}, v_{2}$ and $v_{3}$ are mutually adjacent, and form $C_{3}$, the unique cycle of $U_{M}$. The remaining vertices appear in spiral like disposition with respect to $C_{3}$ starting from $v_{4}$ that is adjacent to $v_{1}$. Moreover, $v_{1}, v_{2}$ and $v_{3}$ are the vertices having the first three largest $\rho$-weight in $U_{M}$.

Let $F_{1}(n, \Delta), F_{2}(n, \Delta)$, and $J_{1}(n, \Delta)$ be the unicyclic graphs with $n$ vertices and the maximum degree $\Delta \geq \frac{n}{2}+1$ as shown in Fig. 3.1. Let $\mathbb{U}(n, \Delta, d)$ be the set of unicyclic graphs on $n$ vertices with the maximum degree $\Delta$ and the second maximum degree $d$. Let $C_{n}$ be the cycle of order $n$.

LEMmA 3.2. If $\Delta-d \geq 2$ and $d \leq n-\Delta$, then

$$
\max \{\rho(U): U \in \mathbb{U}(n, \Delta, d)\}<\max \{\rho(U): U \in \mathbb{U}(n, \Delta, d+1)\}
$$



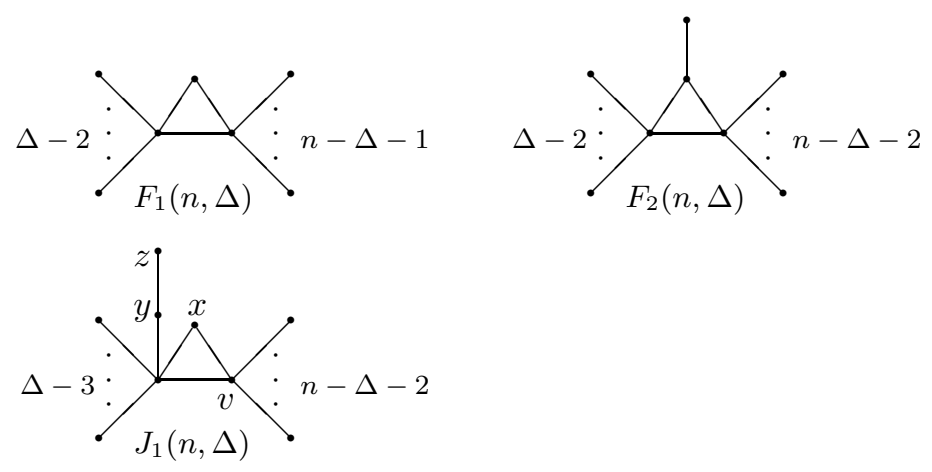

FIG. 3.1. The unicyclic graphs $F_{1}(n, \Delta), F_{2}(n, \Delta)$, and $J_{1}(n, \Delta)$.

Proof. Let $U_{1}$ be a unicyclic graph with the largest spectral radius in $\mathbb{U}(n, \Delta, d)$. Suppose that the degree sequence of $U_{1}$ is $(a)=\left(\Delta, d, d_{3}, \ldots, d_{n}\right)$. Then, $U_{1}$ also has the largest spectral radius in $\Gamma(a)$. Since $\Delta-d \geq 2$, there exists a unique vertex, say $u$, such that $d(u)=\Delta$. Let $f$ be the Perron vector of $U_{1}$. Let $v$ be a vertex of $U_{1}$ such that $f(v)=\max \{f(w): d(w)=d\}$. By Lemmas 2.4 and 3.1 and the choice of $v, u v \in E\left(C_{3}\right)$, where $C_{3}$ is the unique cycle of $U_{M}$. Suppose $V\left(C_{3}\right)=\{u, v, x\}$.

If $d(x) \geq 3$, i.e., there exists some vertex $y$ different from $u, v$ such that $x y \in$ $E\left(U_{1}\right)$, then $y \notin N(v)$. By the choice of $v$ and Lemma 2.4 it follows that $f(v) \geq f(x)$. Let $U^{\prime}=U_{1}+v y-x y$. Then, $U^{\prime} \in \mathbb{U}(n, \Delta, d+1)$. Moreover, by Lemma 2.1 we have $\rho\left(U^{\prime}\right)>\rho\left(U_{1}\right)$.

If $d(x)=2$, since $d \leq n-\Delta$, Lemma 3.1 implies that there exist vertices $y \in N(u)$ $(y \notin\{u, v, x\})$ and $w(w \notin\{u, v, x, y\})$ such that $w \in N(y)$. Then, $w \notin N(v)$. It can be proved similarly with the case of $d(x) \geq 3$.

Remark 3.3. The condition " $d \leq n-\Delta$ " of Lemma 3.2 is necessary. Otherwise, if $d \geq n-\Delta+1$, then $\Delta+d+1 \geq n+2$. Assume that $U$ is a unicyclic graph of $\mathbb{U}(n, \Delta, d+1)$ with $\pi(U)=\left(d_{1}, d_{2}, \ldots, d_{n}\right)$. Then, $d_{3} \geq 2$ and hence $\sum_{i=1}^{n} d_{i} \geq$ $n+2+2+n-3=2 n+1$. So, we arrive at a contradiction because the sum of degrees of vertices pertaining to a unicyclic graph is equal to $2 n$.

Proof of Theorem 1.5 (i). Let $(a)=(\Delta, n-\Delta, 3,1,1, \ldots, 1)$. It is easily checked that $F_{2}(n, \Delta)$ is the unique unicyclic graph in $\Gamma(a)$, and $F_{1}(n, \Delta)$ is the unique unicyclic graph in $\mathbb{U}(n, \Delta, n-\Delta+1)$. Since $d_{2}\left(F_{2}(n, \Delta)\right)<d_{2}\left(F_{1}(n, \Delta)\right)$ and $\Delta-(n-\Delta)=$ $2 \Delta-n \geq 2$, we have $\rho\left(F_{2}(n, \Delta)\right)<\rho\left(F_{1}(n, \Delta)\right)$ by Lemma 3.2. Suppose that the degree sequence of $U$ is $(b)=\left(d_{1}, d_{2}, d_{3}, \ldots, d_{n}\right)$. Since $U \in \mathbb{U}_{n}^{\Delta} \backslash\left\{F_{1}(n, \Delta), F_{2}(n, \Delta)\right\}$, it follows that $d_{1}=\Delta, d_{2} \leq n-\Delta$, and $\Delta \leq n-3$. There are two cases to be considered. 
Case 1. $d_{2}=n-\Delta$.

Since $U \neq F_{2}(n, \Delta)$, we have $(b)=(c)$, where $(c)=(\Delta, n-\Delta, 2,2,1, \ldots, 1)$. Thus, $\rho(U) \leq \rho\left(J_{1}(n, \Delta)\right)$ by Lemma 3.1. Let $f$ be the Perron vector of $J_{1}(n, \Delta)$. Let $v, x, y, z$ be the vertices of $J_{1}(n, \Delta)$ as shown in Fig. 3.1.

If $f(x) \geq f(y)$, let $U^{*}=J_{1}(n, \Delta)+x z-y z$. Then, $U^{*} \cong F_{2}(n, \Delta)$. By Lemma 2.1, we have $\rho\left(J_{1}(n, \Delta)\right)<\rho\left(F_{2}(n, \Delta)\right)$.

If $f(x)<f(y)$, let $U^{*}=J_{1}(n, \Delta)+v y-v x$. Then, $U^{*} \cong F_{2}(n, \Delta)$. By Lemma 2.1, we have $\rho\left(J_{1}(n, \Delta)\right)<\rho\left(F_{2}(n, \Delta)\right)$.

Thus, we can conclude that $\rho(U) \leq \rho\left(J_{1}(n, \Delta)\right)<\rho\left(F_{2}(n, \Delta)\right)$.

Case 2. $d_{2} \leq n-\Delta-1$.

Note that $\Delta-d_{2} \geq \Delta-(n-\Delta-1)=2 \Delta+1-n>2$. By Lemma 3.2 and the proof of Case 1, the result follows.

LEMMA 3.4. If $\Delta \geq \frac{1}{9}(1+\sqrt{6 n+10})^{2}-1$, then $\rho\left(F_{1}(n, \Delta)\right) \leq \sqrt{\Delta+1}$.

Proof. By an elementary computation, we have

$$
\Phi\left(F_{1}(n, \Delta), x\right)=x^{n-4} f_{1}(x)
$$

where $f_{1}(x)=x^{4}-n x^{2}-2 x+(n-\Delta+1) \Delta-n-1$. Thus, $\rho\left(F_{1}(n, \Delta)\right)$ is equal to the maximum root of $f_{1}(x)=0$. When $x \geq \sqrt{\Delta+1}>\sqrt{\frac{n+1}{2}}$, since $f_{1}^{\prime \prime}(x)=12 x^{2}-2 n>$ 0 , it follows that

$$
f_{1}^{\prime}(x)=4 x^{3}-2 n x-2>4\left(\sqrt{\frac{n+1}{2}}\right)^{3}-2 n \sqrt{\frac{n+1}{2}}-2 \geq 0 .
$$

Thus, when $x \geq \sqrt{\Delta+1}>0$, we have

$$
\begin{aligned}
f_{1}(x) & \geq(\Delta+1)^{2}-n(\Delta+1)-2 \sqrt{\Delta+1}+(n-\Delta+1) \Delta-n-1 \\
& \geq 3 \Delta-2 \sqrt{\Delta+1}-2 n \\
& \geq 0
\end{aligned}
$$

Therefore, $\rho\left(F_{1}(n, \Delta)\right) \leq \sqrt{\Delta+1}$.

Proof of Theorem 1.5 (ii). In the proof of this result, we write $\Delta\left(U^{\prime}\right)$ and $\Delta(U)$ as $\Delta^{\prime}$ and $\Delta$, respectively. Set $a=\left\lceil\frac{1}{9}(1+\sqrt{6 n+10})^{2}\right\rceil-1$. Then, $a>\frac{n}{2}+1$. We divide the proof into two cases. 
Case $1 . \Delta^{\prime} \geq a$

Then, Theorem 1.5 (i) and Lemma 3.4 imply that $\rho\left(U^{\prime}\right) \leq \rho\left(F_{1}\left(n, \Delta^{\prime}\right)\right) \leq$ $\sqrt{\Delta^{\prime}+1}$. Since $\Delta>\Delta^{\prime}$ and $U$ has $K_{1, \Delta}$ as its proper subgraph, we can conclude that $\rho(U)>\sqrt{\Delta} \geq \sqrt{\Delta^{\prime}+1} \geq \rho\left(U^{\prime}\right)$.

Case 2. $\Delta^{\prime}<a$.

Then, $\rho\left(U^{\prime}\right)<\rho\left(F_{1}(n, a)\right) \leq \sqrt{a+1}$ follows from Lemmas 2.5 and 3.4 Since $\Delta \geq \frac{1}{9}(1+\sqrt{6 n+10})^{2}$, we have $\Delta \geq\left\lceil\frac{1}{9}(1+\sqrt{6 n+10})^{2}\right\rceil=a+1$. Hence, $\rho(U)>$ $\sqrt{\Delta} \geq \sqrt{a+1}>\rho\left(U^{\prime}\right)$.

4. The proof of Theorem 1.6. In the proof of Theorem 1.6, the next lemma plays a crucial role.

Lemma 4.1. 6] Let $\pi=\left(d_{1}, d_{2}, \ldots, d_{n}\right)$ be a non-increasing degree sequence, where $d_{1} \geq d_{2} \geq 3$ and $d_{n}=1$. Let $B_{M}$ be a bicyclic graph with the largest spectral radius in $\Gamma(\pi)$, and $\left\{v_{1}, v_{2}, \ldots, v_{n}\right\}$ be the vertices of $B_{M}$ with $d\left(v_{i}\right)=d_{i}$. Then, $B_{M}$ contains $B^{*}$ as its induced subgraph, which is formed by adding an edge to two non-incident vertices of $C_{4}$ such that $v_{1}$ and $v_{2}$ are the two vertices of $B^{*}$ of degree $3, v_{3}$ and $v_{4}$ are the two vertices of $B^{*}$ of degree 2 . The remaining vertices appear in spiral like dispositions with respect to $B^{*}$ starting from $v_{5}$ that is adjacent to $v_{1}$. Moreover, $v_{1}, v_{2}, v_{3}$ and $v_{4}$ are the vertices having the first four largest $\rho$-weight in $B_{M}$.
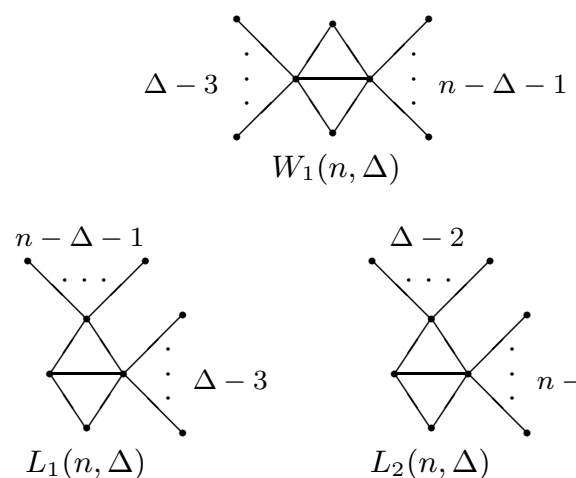

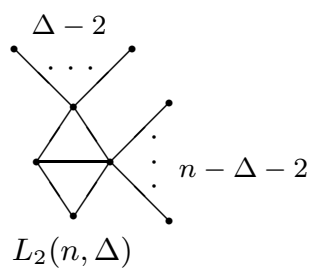

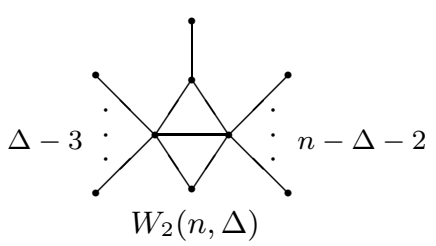

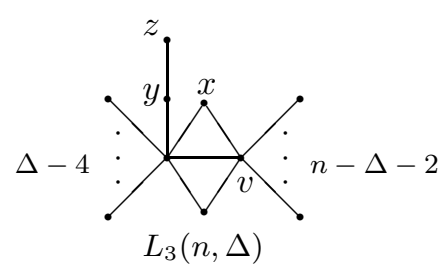

FIG. 4.1. The bicyclic graphs $W_{1}(n, \Delta), W_{2}(n, \Delta), L_{1}(n, \Delta), L_{2}(n, \Delta) n$ and $L_{3}(n, \Delta)$.

Let $W_{1}(n, \Delta), W_{2}(n, \Delta), L_{1}(n, \Delta), L_{2}(n, \Delta)$, and $L_{3}(n, \Delta)$ be the bicyclic graphs with $n$ vertices and the maximum degree $\Delta \geq \frac{n+3}{2}$ as shown in Fig. 4.1]

Let $\mathbb{B}(n, \Delta, d)$ be the set of bicyclic graphs on $n$ vertices with the maximum degree 
$\Delta$ and the second maximum degree $d$. If $y \in N(x)$ and $d(y)=1$, then we call $y$ a pendant neighbor of $x$. If $d(x) \geq 2$, then we call $x$ a non-pendant vertex.

LEMMA 4.2. If $\Delta-d \geq 2$ and $d \leq n-\Delta+1$, then

$$
\max \{\rho(B): B \in \mathbb{B}(n, \Delta, d)\}<\max \{\rho(B): B \in \mathbb{B}(n, \Delta, d+1)\} .
$$

Proof. Let $B_{1}$ be a bicyclic graph with the largest spectral radius in $\mathbb{B}(n, \Delta, d)$. Then, $B_{1}$ also has the largest spectral radius in $\Gamma(\pi)$, where $\pi=\pi\left(B_{1}\right)$. Since $\Delta-d \geq 2$, there exists a unique vertex, say $u$, such that $d(u)=\Delta$. Let $f$ be the Perron vector of $B_{1}$. Let $v$ be a vertex of $B_{1}$ such that $f(v)=\max \{f(w): d(w)=d\}$. There are two cases to be considered.

Case 1. $u v \notin E\left(B_{1}\right)$.

Let $P_{u v}$ be the shortest path of $B_{1}$ from $u$ to $v$, and let $x$ be the vertex in $P_{u v}$ adjacent to $u$. Let $B^{\prime}=B_{1}+u v-u x$. Then, $B^{\prime} \in \mathbb{B}(n, \Delta, d+1)$. On the other hand, by Lemma 2.4 and the choice of $v, f(v) \geq f(x)$. By Lemma 2.1. we can conclude that $\rho\left(B^{\prime}\right)>\rho\left(B_{1}\right)$.

Case 2. $u v \in E\left(B_{1}\right)$.

In the following, let $x$ denote a non-pendant vertex different from $u, v$, and $P_{v x}$ be the shortest path of $B_{1}$ from $v$ to $x$.

Subcase 2.1. There exist vertices $x$ and $y$, such that $y \in N(x) \backslash N(v)$ and $y$ is not in $P_{v x}$.

By the choice of $v$ and Lemma 2.4, it implies that $f(v) \geq f(x)$. Let $B^{\prime}=B_{1}+$ $v y-x y$. Then, $B^{\prime} \in \mathbb{B}(n, \Delta, d+1)$. Moreover, by Lemma 2.1 we have $\rho\left(B^{\prime}\right)>\rho\left(B_{1}\right)$.

Subcase 2.2. For every non-pendant vertex $x$, if $y \in N(x)$ and $y$ is not in $P_{v x}$, then $y \in N(v)$.

Thus, we can conclude that if $w$ is a non-pendant vertex different from $u, v$, then $w$ has no pendant neighbors. Next we shall prove that $d \geq 3$.

By the hypothesis, $B_{1}$ has non-pendant vertices $x$, and $y$ such that $y \in N(v) \cap$ $N(x)$ and $y$ is not in $P_{v x}$. If $y \neq u$, then $d \geq 3$ because $u v \in E\left(B_{1}\right)$. If $y=u$, since $B_{1}$ is a bicyclic graph, there is at least one vertex $z(z \notin\{u, v, x\})$ such that $d(z) \geq 2$. Let $P_{v z}$ be the shortest path of $B_{1}$ from $v$ to $z$. Then, there is at least one vertex $z_{1}$ such that $z_{1}$ is not in $P_{v z}$ and $z_{1} \in N(v) \cap N(z)$. Note that $d$ is the second maximum degree of $B_{1}$. Then, $d \geq 3$. 
Since $\Delta-d \geq 2$ and $d \geq 3, B_{1}$ has at least a pendant vertex because the sum of degrees of vertices of $B_{1}$ is equal to $2 n+2$. By Lemma 4.1, we can conclude that $B_{1} \cong W_{1}(n, \Delta)$. It is a contradiction to $d \leq n-\Delta+1$. Thus, Subcase 2.2 cannot appear.

REMARK 4.3. The condition " $d \leq n-\Delta+1$ " of Lemma 4.2 is necessary. Otherwise, if $d \geq n-\Delta+2$, then $\Delta+d+1 \geq n+3$. Assume that $B$ is a bicyclic graph of $\mathbb{B}(n, \Delta, d+1)$ with $\pi(B)=\left(d_{1}, d_{2}, \ldots, d_{n}\right)$. Then, $d_{3} \geq d_{4} \geq 2$ and hence $\sum_{i=1}^{n} d_{i} \geq n+3+4+n-4=2 n+3$. So, we arrive at a contradiction because the sum of degrees of vertices pertaining to a bicyclic graph is equal to $2 n+2$.

Proof of Theorem 1.6 (i). It is easily checked that $W_{1}(n, \Delta)$ is the unique bicyclic graph in $\mathbb{B}(n, \Delta, n-\Delta+2)$. Since $d_{2}\left(W_{2}(n, \Delta)\right)<d_{2}\left(W_{1}(n, \Delta)\right)$ and $\Delta-$ $d_{2}\left(W_{2}(n, \Delta)\right)=\Delta-(n-\Delta+1)=2 \Delta-1-n \geq 2$, we have $\rho\left(W_{2}(n, \Delta)\right)<\rho\left(W_{1}(n, \Delta)\right)$ by Lemma 4.2. Suppose that the degree sequence of $B$ is $(a)=\left(d_{1}, d_{2}, d_{3}, \ldots, d_{n}\right)$. Since $B \in \mathbb{B}_{n}^{\Delta}$, we have $d_{1}=\Delta \geq d_{2} \geq d_{3} \geq d_{4} \geq 2$. Recall that $B \in \mathbb{B}_{n}^{\Delta} \backslash\left\{W_{1}(n, \Delta)\right.$, $\left.W_{2}(n, \Delta)\right\}$. Then, $d_{2} \leq n-\Delta+1$. Let $(b)=(\Delta, n-\Delta+1,3,2,1,1, \ldots, 1)$. There are two cases to be considered.

Case 1. $d_{2}=n-\Delta+1$.

Subcase 1.1. $(a)=(b)$.

Note that $W_{2}(n, \Delta), L_{1}(n, \Delta)$, and $L_{2}(n, \Delta)$ are precisely all the bicyclic graphs in $\Gamma(b)$. Since $B \nsubseteq W_{2}(n, \Delta), B$ is isomorphic with $L_{1}(n, \Delta)$ or $L_{2}(n, \Delta)$. By Lemma 4.1, $\rho\left(L_{2}(n, \Delta)\right)<\rho\left(W_{2}(n, \Delta)\right)$ and $\rho\left(L_{1}(n, \Delta)\right)<\rho\left(W_{2}(n, \Delta)\right)$.

Subcase 1.2. $(a) \neq(b)$.

Then, $(a)=(c)$, where $(c)=(\Delta, n-\Delta+1,2,2,2,1, \ldots, 1)$. By Lemma 4.1 , $\rho(B) \leq \rho\left(L_{3}(n, \Delta)\right)$. Let $v, x, y$, and $z$ be the vertices of $L_{3}(n, \Delta)$ as shown in Fig. 4.1. Let $f$ be the Perron vector of $L_{3}(n, \Delta)$.

If $f(x) \geq f(y)$, let $B_{1}=L_{3}(n, \Delta)+x z-y z$. Then, $B_{1} \cong W_{2}(n, \Delta)$. By Lemma 2.1. we have $\rho\left(L_{3}(n, \Delta)\right)<\rho\left(B_{1}\right)=\rho\left(W_{2}(n, \Delta)\right)$.

If $f(x)<f(y)$, let $B_{1}=L_{3}(n, \Delta)+v y-v x$. Then, $B_{1} \cong W_{2}(n, \Delta)$. By Lemma 2.1. we have $\rho\left(L_{3}(n, \Delta)\right)<\rho\left(B_{1}\right)=\rho\left(W_{2}(n, \Delta)\right)$.

Case 2. $d_{2} \leq n-\Delta$.

Note that $\Delta-d_{2} \geq \Delta-(n-\Delta)=2 \Delta-n>2$. Then, $\rho(B)<\rho\left(W_{2}(n, \Delta)\right)$ by Lemma 4.2 and the proof of Case 1 . 
LEMMA 4.4. If $\Delta \geq \frac{1}{9}(2+\sqrt{6 n+28})^{2}-1$, then $\rho\left(W_{1}(n, \Delta)\right) \leq \sqrt{\Delta+1}$.

Proof. By an elementary computation, we have

$$
\Phi\left(W_{1}(n, \Delta), x\right)=x^{n-4} f_{2}(x)
$$

where $f_{2}(x)=x^{4}-(n+1) x^{2}-4 x+(n-\Delta+2) \Delta-n-5$. When $x \geq \sqrt{\Delta+1}>\sqrt{\frac{2 n}{3}+3}$, since $f_{2}^{\prime \prime}(x)=12 x^{2}-2 n-2>0$, we have

$$
f_{2}^{\prime}(x)=4 x^{3}-(2 n+2) x-4>4\left(\sqrt{\frac{2 n}{3}+3}\right)^{3}-(2 n+2) \sqrt{\frac{2 n}{3}+3}-4>0 .
$$

Thus, when $x \geq \sqrt{\Delta+1}>0$, it follows that

$$
\begin{aligned}
f_{2}(x) & \geq(\Delta+1)^{2}-(n+1)(\Delta+1)-4 \sqrt{\Delta+1}+(n-\Delta+2) \Delta-n-5 \\
& =3 \Delta-5-2 n-4 \sqrt{\Delta+1} \\
& \geq 0
\end{aligned}
$$

Therefore, $\rho\left(W_{1}(n, \Delta)\right) \leq \sqrt{\Delta+1}$.

Proof of Theorem 1.6 (ii). In the proof of this result, we write $\Delta\left(B^{\prime}\right)$ and $\Delta(B)$ as $\Delta^{\prime}$ and $\Delta$, respectively. Set $b=\left\lceil\frac{1}{9}(2+\sqrt{6 n+28})^{2}\right\rceil-1$. Then, $b>\frac{n+3}{2}$. We divide the proof into the following two cases.

Case $1 . \Delta^{\prime} \geq b$.

Then, Theorem 1.6 (i) and Lemma 4.4 imply that $\rho\left(B^{\prime}\right) \leq \rho\left(W_{1}\left(n, \Delta^{\prime}\right)\right) \leq$ $\sqrt{\Delta^{\prime}+1}$. Since $\Delta>\Delta^{\prime}$ and $B$ has $K_{1, \Delta}$ as its proper subgraph, we can conclude that $\rho(B)>\sqrt{\Delta} \geq \sqrt{\Delta^{\prime}+1} \geq \rho\left(B^{\prime}\right)$.

Case 2. $\Delta^{\prime}<b$.

Then, $\rho\left(B^{\prime}\right)<\rho\left(W_{1}(n, b)\right) \leq \sqrt{b+1}$ follows from Lemmas 2.5 and 4.4. Since $\Delta \geq \frac{1}{9}(2+\sqrt{6 n+28})^{2}$, it follows that $\Delta \geq\left\lceil\frac{1}{9}(2+\sqrt{6 n+28})^{2}\right\rceil=b+1$. Thus, $\rho(B)>\sqrt{\Delta} \geq \sqrt{b+1}>\rho\left(B^{\prime}\right)$.

Acknowledgment. The authors would like to thank the anonymous referee for his valuable comments and suggestions, which led to an improvement of the original manuscript. 


\section{REFERENCES}

[1] F. Belardo, E.M. Li Marzi, S.K. Simić, and J.F. Wang. On the spectral radius of unicyclic graphs with prescribed degree sequence. Linear Algebra Appl., 432: 2323-2334, 2010.

[2] T. Blyıkoğlu and J. Leydold. Graphs with given degree sequence and maximal spectral radius. Electron. J. Combin., 15:R119, 9 pp., 2008.

[3] D.M. Cvetković. Some possible directions in further investigations of graph spectra. Algebraic methods in graph theory, Vol. I, II (Szeged, 1978), Colloq. Math. Soc. János Bolyai, 25:47-67, North-Holland, Amsterdam-New York, 1981.

[4] A.J. Hoffman and J.H. Smith. On the spectral radii of topologically equivalent graphs. In M. Fiedler (editor), Recent Adances in Graph Theory, Academia Praha, New York, 1975.

[5] W.S. Lin and X.F. Guo. Ordering trees by their largest eigenvalues. Linear Algebra Appl., 418:450-456, 2006.

[6] Y.L. Liu and B.L. Liu. The spectral radius of bicyclic graphs with prescribed degree sequences. Linear Algebra Appl., 433:1015-1023, 2010.

[7] M.H. Liu and B.L. Liu. On the spectral radii and the signless Laplacian spectral radii of $c$-cyclic graphs with fixed maximum degree. Linear Algebra Appl., 435:3045-3055, 2011.

[8] B.F. Wu, E.L. Xiao, and Y. Hong. The spectral radius of trees on $k$ pendant vertices. Linear Algebra Appl., 395:343-349, 2005.

[9] X.Y. Yuan and Y. Chen. Some results on the spectral radii of bicyclic graphs. Discrete Math., 310:2835-2840, 2010.

[10] X.Y. Yuan, H.Y. Shan, and B.F. Wu. On the spectral radius of unicyclic graphs with fixed maximum degree. Ars Combin., CII:21-31, 2011. 